\title{
Effects of percutaneous stimulation on motor unit firing behavior in man
}

\author{
Y. Masakado*, G. Kamen**, and C.J. De Luca \\ Neuromuscular Research Center, Boston, University, Boston MA 02215, USA
}

Received November 20, 1990 / Accepted May 10, 1991

Summary. Motor unit firing behavior in human first dorsal interosseous (FDI) muscle was studied during controlled constant force isometric contractions. The threshold at which motor units were recruited and the mean firing rate at $50 \%$ of maximal voluntary contraction (MVC) were evaluated following stimulation of the skin area over the second digit. Stimulation of cutaneous receptors tended to increase the recruitment threshold of most of the motor units recruited under $20 \%$ MVC, while high-threshold motor units (those recruited over $30 \%$ MVC) generally exhibited a decrease in recruitment threshold. Less dramatic changes in motor unit firing rates were observed, but those motor units recruited over $30 \%$ MVC exhibited some increase in firing rate. The relationship between the change in recruitment threshold and change in motor unit firing rate is not rigid and seems to be susceptible to considerable synaptic noise.

Key words: Motor units - Cutaneous receptors - Size principle - Motoneuron - Recruitment threshold - Man

\section{Introduction}

A basic tenet of motor control, the size principle of motoneuron recruitment (Henneman et al. 1965), was proposed a quarter-century ago. This theory states that, within any given muscle, motoneurons are activated in a fixed order according to increasing size. Smaller motoneurons innervating siow-twitch motor units (MUs) are recruited at low force thresholds, while larger motoneurons innervating fast-twitch motor units are recruited at increasing force intensity. A corollary idea also suggests that derecruitment or motor unit deactivation occurs in

Present addresses: * School of Medicine, Keio University, Tokyo, Japan

** Department of Physical Therapy, Boston University, 635 Commonwealth Avenue, Boston, MA 02215, USA

offprint requests to: G. Kamen (address see footnote) a reverse order (Clamann et al., 1974, 1976). A number of both human and animal studies have demonstrated the generality of the theory under a variety of conditions (cf. Binder and Mendell 1990).

There have been numerous challenges to the size principle and some exceptions to the theory have been noted, perhaps indicative of the multitude of factors controlling motoneuron excitability. For example, Desmedt and Godaux (1981) report some rank reversal when the first dorsal interosseous (FDI) acts as an index finger flexor rather than an abductor. They suggested that the pattern of activation may well be different when a muscle operates as a prime mover rather than a synergist. Similar recruitment reversals were obtained in other muscles including the abductor pollicis brevis (Thomas et al. 1987), extensor digitorum (Thomas et al. 1978), mentalis (McLean 1984) and the biceps brachii (Ter Haar Romeny et al. 1982). Nardone et al. (1989) have presented evidence suggesting that some selective activation of high-threshold motor units may be possible during lengthening contractions. Alterations in recruitment order have been observed in other experiments involving non-isometric contractions (Person 1974). These reversals in recruitment order are thought to be due to the influence of a variety of synaptic inputs.

There are some advantages for motor units with larger twitch forces to be recruited early during a contraction, especially under conditions requiring a rapid movement. For example, in some situations activation of cutaneous mechanoreceptors or other nociceptors should be followed by the fastest possible movement. There is evidence in the cat that low-threshold cutaneous afferents can preferentially facilitate fast-twitch motor units (Burke 1973; Burke et al. 1970). Cutaneous electrical stimulation can produce a recruitment order reversal in the medial gastrocnemius muscle of the decerebrate cat (Kanda et al. 1977), and similar results have been obtained in $\mathrm{m}$. lumbricalis in anesthetized animals (Mizote 1982). These observations suggest that the distribution of cutaneous input to motoneurons may be dissimilar to that of la afferents. 
Cutaneous afferents may well have a more important role in motor control (Burke et al. 1989; Sabbahi and De Luca 1982) than previously recognized. In man, skin stimulation has been reported to produce a reversal in recruitment order, as well as changes in the threshold force at which a motor unit is recruited (Garnett and Stephens 1979). Similar effects were obtained using active touch (Kanda and Desmedt 1983). Recruitment reversals have also been noted following partial ischemic or lidocaine blockade and local muscle cooling (Grimby and Hannerz 1976). Moreover, changes in motor unit firing rates have also been observed with skin stimulation (Datta and Stephens 1979, 1981).

However, the evidence supporting an alteration in recruitment order from skin afferents remains equivocal. Inasmuch as some reports have indicated that no recruitment reversal is obtained by skin stimulation (Stein and Bertoldi 1981), we endeavored to re-examine the issue. Previous reports concerning the role of cutaneous receptors on motor unit firing behavior have been predominantly confined to low-threshold motor units. The present study contains observations made from motor units activated at forces ranging up to $40 \%$ of maximal effort.

\section{Material and methods}

\section{Subjects}

Rocordings were made from the first dorsal interosseous (FDI) muscle in seven young subjects (ages 20-32 years) with no known neurological disorders. Informed consent was obtained from all individuals.

\section{Signal acquisition and identification}

Motor unit action potential trains (MUAPTs) were obtained by utilizing the myoelectric (ME) signal acquisition and decomposition technique as detailed in earlier papers (Mambrito and De Luca 1983, 1984; Stashuk and De Luca 1989). A quadrifilar electrode was used to record ME activity during active isometric muscle contraction using a bandpass of $1 \mathrm{kHz}$ to $10 \mathrm{kHz}$. The resulting three channels of $\mathrm{ME}$ information were used to identify individual motor unit action potential (MUAP) firings from the composite myoelectric signal. Decomposition of the signal into individual motor unit action potential trains was performed by an operator-interactive program utilizing both template-matching and firing history statistics. The algorithm also identified motor unit firing oocurrences when two or motor units fired at precisely the same time. The decomposition procedure allowed us to identify each motor unit firing with an accuracy close to 100\%. Typical motor unit recruitment and discharge behavior information obtained by the decomposition technique is displayed in Fig. 1.

The subjects were seated with the right hand inserted in a molded restraining device and the index finger placed between the mold and a force transducer. This device served to isolate the force produced from the FDI muscle.

\section{Skin stimulation of the index finger}

Two ring electrodes smeared with electrode jelly were placed around the index finger between the proximal interphalangeal joint and metacarpophalangeal joint. Interelectrode distance was about two $\mathrm{cm}$. Square wave stimuli were delivered by a constant voltage stimulator at a frequency of $50 \mathrm{~Hz}$ using a pulse width of $0.1 \mathrm{~ms}$. Stimulus strength was set at three times threshold for perception. Perceptual stimulation threshold was measured before each experiment.

\section{Experimental procedure}

The subject's maximal voluntary contraction (MVC) during index finger abduction was determined at the beginning of the experiment. The needle was then positioned into a suitable recording site, while the subject maintained a mild contraction. A line trajectory was then displayed on a computer screen. This trajectory required the subject to perform a smooth isometric contraction consisting of an initial increase in force to $50 \%$ MVC over a five-s interval, maintaining the $50 \%$ MVC contraction for $10 \mathrm{~s}$, and a five-s period of smoothly decreasing force output to zero. A sample force trace is plotted in Fig. 1.

The index finger was then stimulated at three times threshold for peroeption. After a three-minute stimulation interval, the contraction and data collection protocol were repeated while stimulation was maintained. A minimal 10-min recovery period was allotted before the needle was repositioned and the protocol repeated. Recent reports have suggested that recruitment threshold may decrease with repeated contraction (Suzuki et al. 1988, 1989, 1990). In order to eliminate the possibility that our results might be due to some repeated contraction effoct, we performed three control
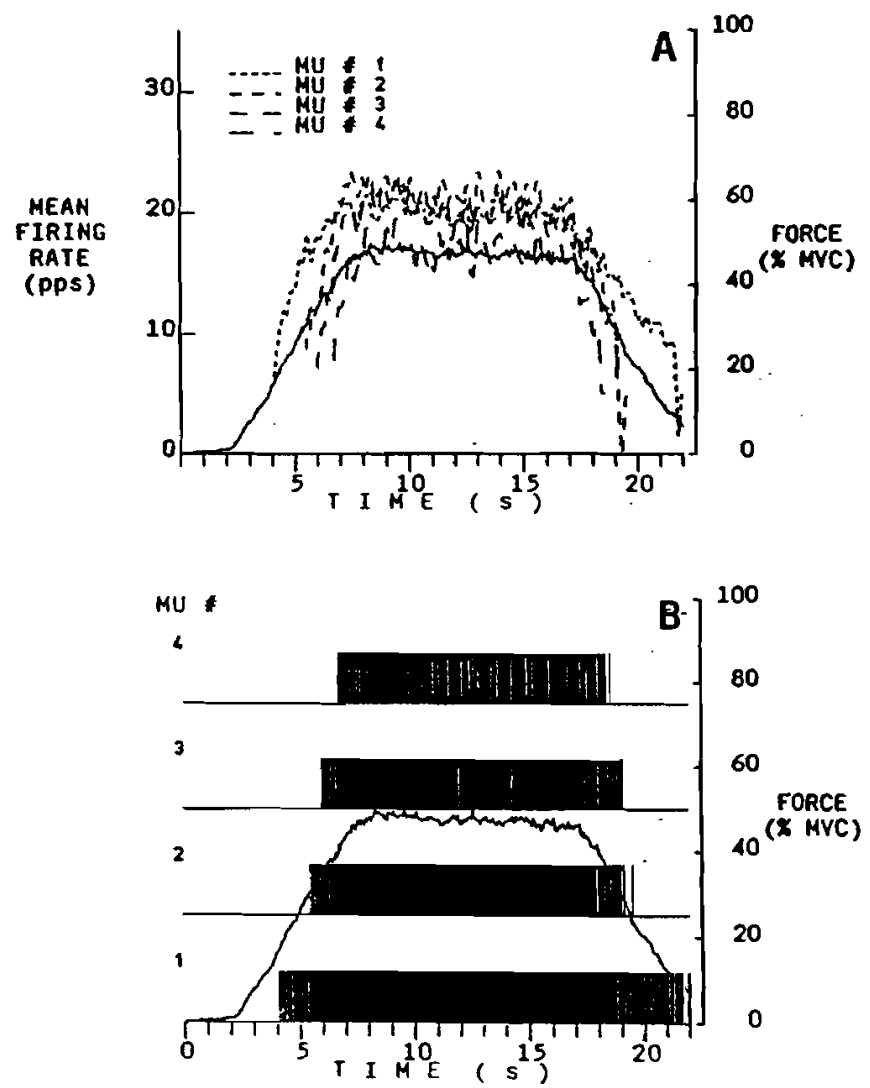

Fig. 1. Firing behavior of four concurrently active motor units are shown superimposed on the force output (solid line) recorded during isometric contraction of the FDI. The force level is given in percent of maximal voluntary contraction (MVQ) on the right ordinate. In (A), the firing rates of the four motor unis are shown, while individual firing histories are plotted in (B) 
experiments in which repeated contractions were performed, separated by a three-minute interval. These control experiments were performed on separate days.

\section{Determination of motor unit recruitment thresholds and mean firing rates}

Motor unit recruitment force thresholds and mean firing rates at 50\% MVC were estimated for the MUs successfully tracked throughout both contractions. The recruitment threshold was determined as the mean force level computed over the first five discharges of the MU. This arbitrary method of computing the force at which a motor unit is activated reduces the variability inherent in MU recruitment threshold measurements and provides an estimate of the force level when the unit was actually contributing mechanically to the overall muscular force. The mean firing rate at 50\% MVC was estimated for each motor unit by taking the inverse of the mean interpulse interval during the first five seconds of the plateau portion of the contraction.

\section{Results}

\section{Reliability of motor unit identification}

To ensure that the same motor units were being studied during both contractions (pre- and post-stimulation), the shapes of the MUAPs of each motor unit were studied to confirm that the position of the needle relative to the surrounding muscle fibers was stable and therefore the same motor units were followed. Examples of individual MUAP templates followed during the experiment are shown in Fig. 2. This figure demonstrates the typical similarity of MUAP shapes on three channels that was required before the assumption that the same motor unit was being tracked throughout both contractions was accepted. When it was not clear whether a particular motor unit could be followed, the experimental data were discarded. Of 25 experiments conducted, 11 experiments contained data in which every firing occurrence of at least one motor unit could be identified in two different contractions. These 11 experiments, from seven subjects, yielded data from 52 motor units recorded in 25 pairs of contractions. On average, two motor units were identified in each contraction (range: 1-4 motor units).

One important methodological question concerns the accuracy of identifying motor unit firing occurrences, especially during two successive contractions at $50 \%$ MVC with an interval of several minutes between contractions. It has been previously demonstrated that the decomposition technique used can accurately identify several motor unit action potential trains in an active contraction (LeFever et al. 1982; Mambrito and De Luca

\section{PRE STIMULATION}

\section{POST STIMULATION}

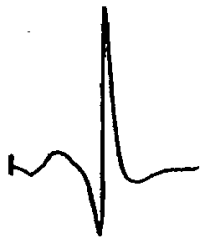

MU \#1

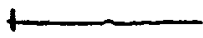

L

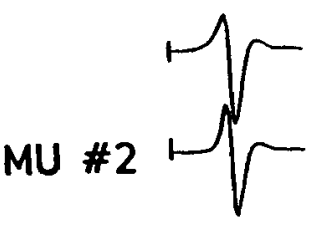

$\sqrt{ }$
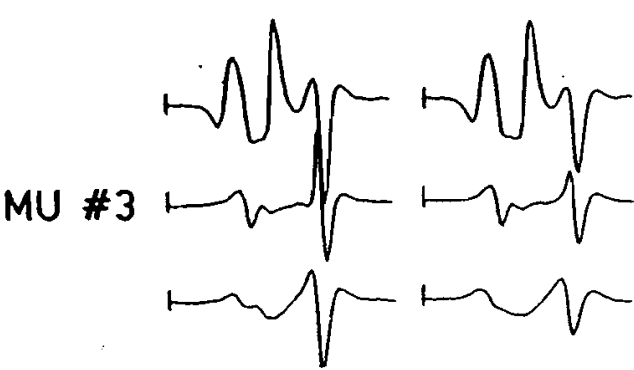
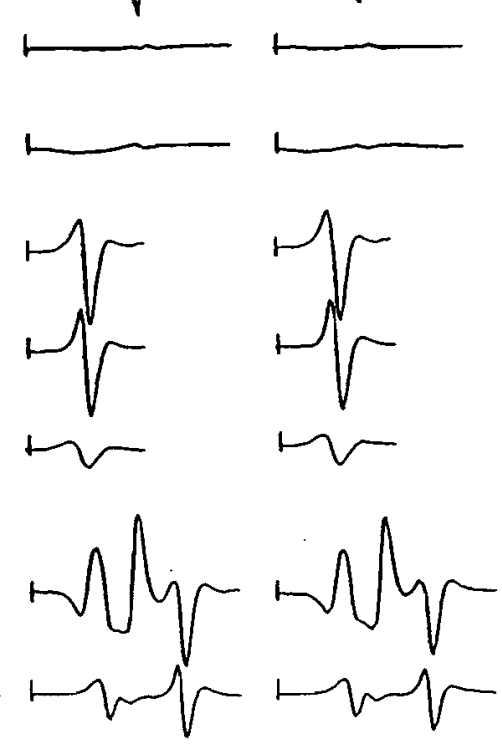

$\sim \sqrt{ }$
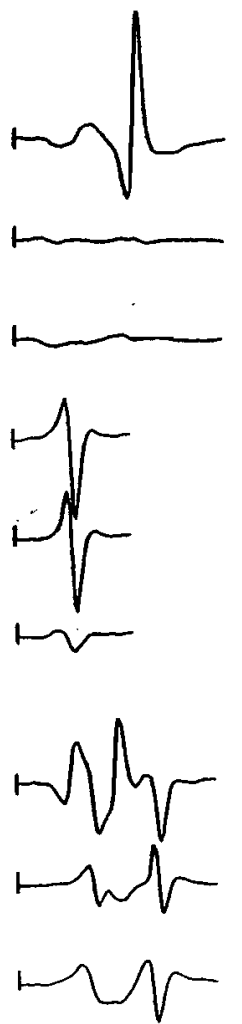
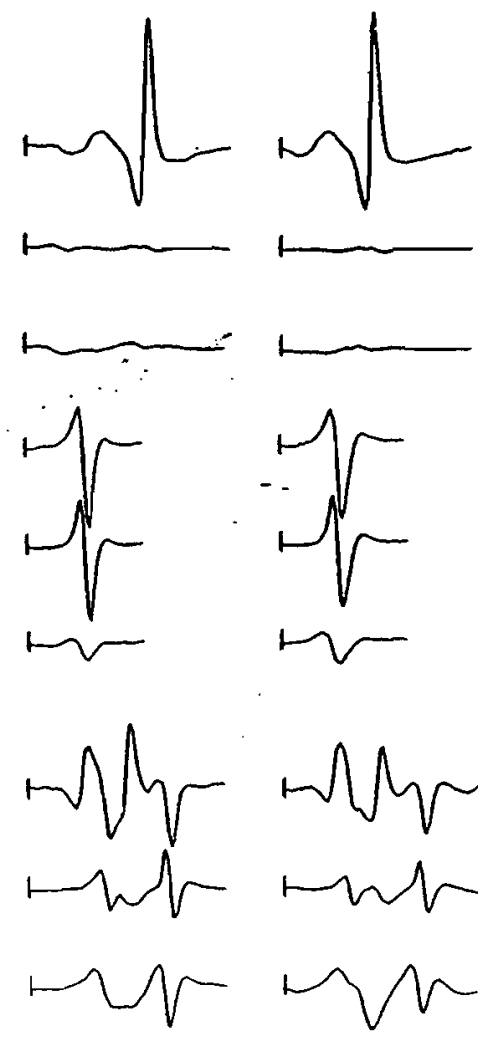

Fig. 2. Typical motor unit templates obtained from three motor units during the pre- and post-stimulation contractions. The three different views of the motor unit provided by the three-channel recording facilitates unit discrimination. The shape of MU no3 was somewhat variable, but the highly polyphasic shapes enabled unit identification 


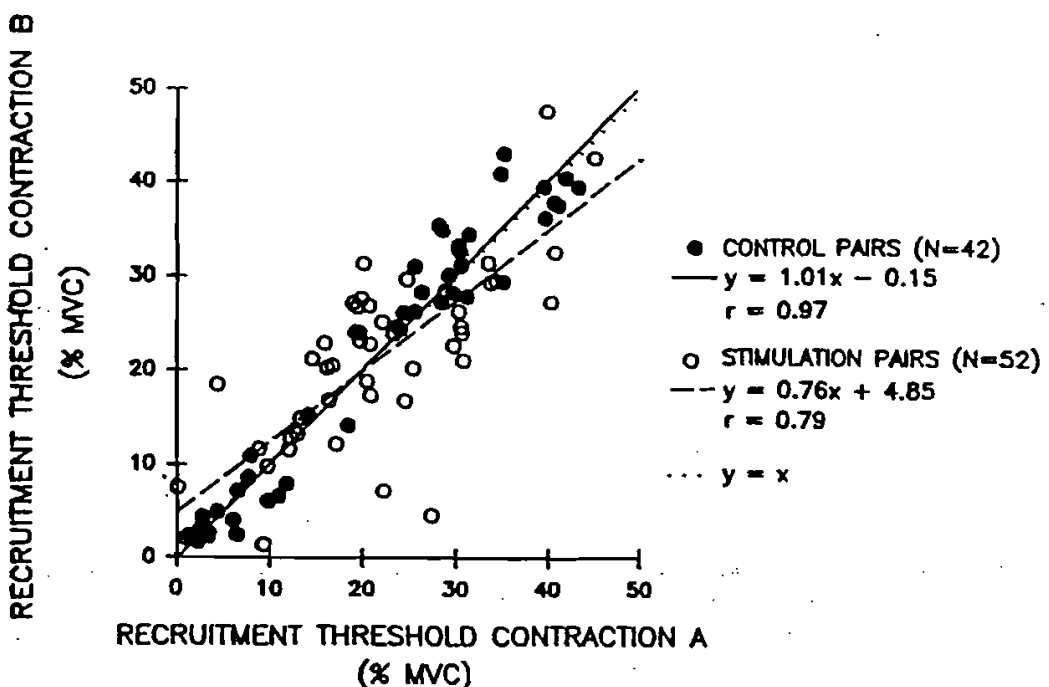

Flg. 3. Changes in recruitment threshold during skin stimulation and control experiments. For the skin stimulation experiments, the recruitment threshold observed during the first contraction are plotted on the abscissa against the threshold observed during the second, post-stimulation contraction (plotted on the ordinate). For the control experiments, the abscissa and ordinate points refer to the first and second contractions, respectively. For reference, the unity slope line $(x=y)$ has also been plotted. Most of the low-

1984). Our records (see Fig. 2) indicate that even units with polyphasic shapes can be recorded at these force levels, so long as one has multiple views of the action potential.

\section{Effect of skin stimulation on motor unit recruitment threshold}

The skin stimulation produced a change in the threshold force at which motor units were recruited during a voluntary contraction (Figs. 3 and 4). Twenty-one of the 25 motor units recruited under $20 \%$ MVC exhibited an increase in the threshold force for recruitment, while recruitment threshold decreased in the remaining four motor units. Of the 16 motor units recruited between $20 \%$ and $30 \%$ MVC, eight increased recruitment threshold, while the other eight manifested a decrease in the threshold force value. Ten of the 11 motor units recruited over 30\% MVC decreased in threshold force for recruitment. A chi-square test revealed that the frequencies in each of these six cells (see Fig. 4) were different than expected by chance occurrence $\left(\chi^{2}=28.7, \mathrm{df}=5\right.$; $p<0.05$ ). Thus, the threshold of most of the motor units recruited under $20 \%$ MVC increased with electrical stimulation, while the threshold of those recruited over $30 \%$ MVC decreased. Mixed results were obtained for motor units with an initial recruitment threshold of $20 \%-30 \%$ MVC.

\section{Control experiments}

Control observations were made in three subjects. These experiments yielded 42 pairs of contractions (two con-

threshold units (below 20\% MVC) increased in recruitment threshold with stimulation, while almost all of the high-threshold units (above 30\% MVC) decreased recruitment threshold with skin stimulation. For the motor units whose recruitment thresholds were assessed before and after a three-minute rest interval, the values obtained for the slope of the regression line (1.01) and the $y$-intercept $(-0.15)$ indicate that repeated effort had little effect on motor unit recruitment threshold

tractions separated by a three-min rest interval) in which 24 motor units were identified. During the same experiment, some motor units were identified in three or more contractions, each contraction separated by a three-min interval. In the skin stimulation experiments, the Pearson correlation coefficient between recruitment threshold obtained prior to stimulation and that observed after the three-min stimulation was $r=0.79$. In the control experiments, recruitment threshold from the first and second contractions correlated $r=0.97$. The difference between these two correlation coefficients was statistically significant $(z=5.71 ; p<0.05)$ and likely reflects some variability in motoneuron excitability produced by the stimula-

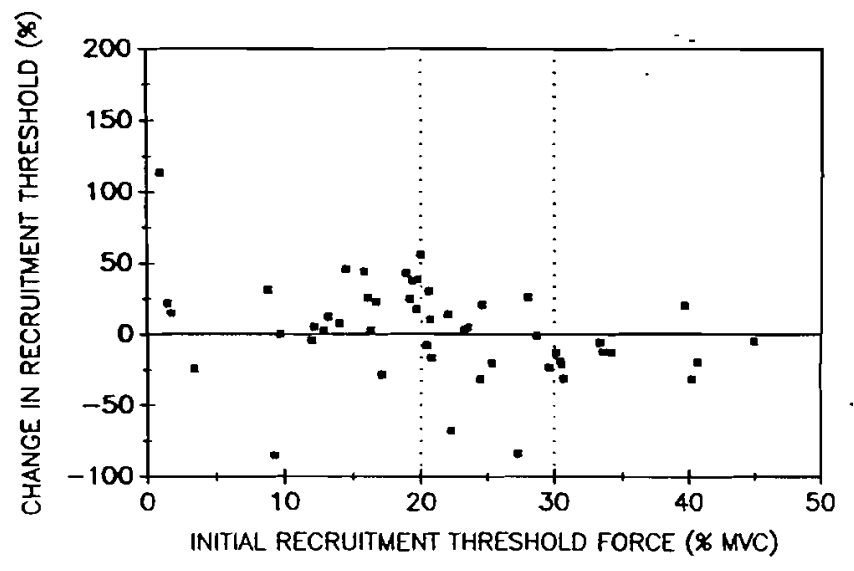

Fig. 4. Relative change in motor unit recruitment threshold observed as a result of the electrical stimulation protocol are plotted as a function of initial recruitment threshold. Two moter units which increased in threshold over $200 \%$ (both low-threshold units) are not represented in this figure 


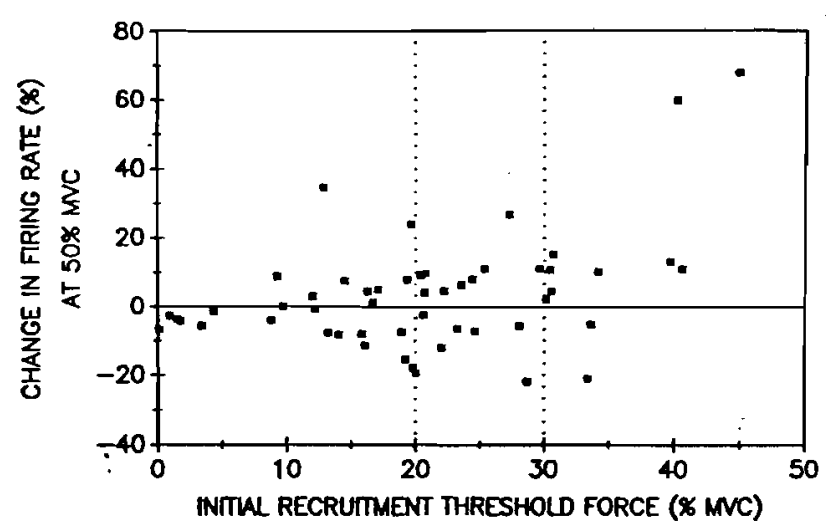

Fig. 5. Relative changes in motor unit firing rate following electrical stimulation as a function of initial recruitment threshold. Vertical dotted lines have been plotted at the 20\% MVC and 30\% MVC points

tion protocol. The slope of the regression line for recruitment threshold was very close to unity $(\beta=1.01)$ in the control experiments, but was significantly lower $(F(1,90)=8.21 ; p<0.05)$ in the stimulation experiments (see Fig. 3). Thus, our results provide little support for a change in recruitment threshold as a result of repeated effort, and the changes produced by skin stimulation seem to be unrelated to repeated measurement issues.

\section{Effect of skin stimulation on motor unit firing rates}

The skin stimulation produced more subtle changes in motor unit firing rate than those observed for recruitment threshold. Linear regression analysis revealed that there was a slight trend towards higher firing rates for the motor units recruited above $30 \% \mathrm{MVC}$, and lower firing rates for the low-threshold motor units $(r=0.42, p<0.05$; see Fig. 5). Fourteen of the 25 motor units recruited under 20\% MVC decreased their firing rate. The stimulation produced firing rate increases in about half $(8 / 14)$ of the motor units recruited between 20 and $30 \% \mathrm{MVC}$, and in eight of the eleven units recruited over $30 \%$ MVC. (Note that it was not always possible to obtain both recruitment threshold and firing rate for each motor unit, due to instability in the position of the recording electrode). Chi-square analysis revealed that the distribution of frequencies in these categories (Fig. 5) was not significantly different from that expected by chance $\left(\chi^{2}=10.96\right.$, $\mathrm{df}=5 ; p=0.052$ ).

There was a slight tendency $(r=0.38 ; p<0.05)$ for the motor units that exhibited the greatest change in recruitment threshold to also display the largest change in firing rate (Fig. 6). A chi-square analysis, conducting using the four cells shown in Fig. 6 revealed that the frequencies in these cells was significantly different that expected by chance occurrence alone $\left(x^{2}=11.2, \mathrm{df}=3 ; p<0.05\right)$. So it does seem that a change in recruitment threshold is accompanied by some change in motor unit firing rate. A similar chi-square analysis for the control data re-

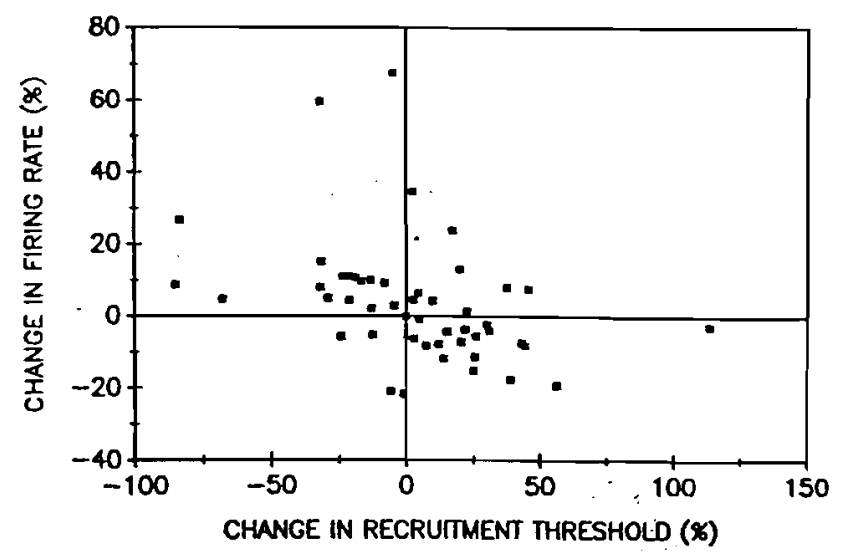

Fig. 6. The relationship between changes in motor unit recruitment threshold and corresponding changes in firing rate following eloctrical stimulation. Those units which tended to decrease in recruitment threshold exhibited an increase in firing rate. Correspondingly, the motor units which increased in recruitment threshold mostly tended to decrease in firing rate

vealed no change in cell frequencies $\left(x^{2}=4.18, \mathrm{df}=5\right.$; $p>0.05$ ), indicating that in the control experiments motor unit firing rates were unaffected by a three-min rest interval.

\section{Discussion}

The results demonstrate that stimulation of skin receptors changes motor unit recruitment threshold and firing rate in a clearly differential manner. In the higherthreshold (and presumably larger) motor units recruited above 30\% MVC, additional input from cutaneous receptors results in a predominant decrease in recruitment threshold and an increase in firing rate. Conversely, the smaller, lower-threshold motor units tend to exhibit an increase in recruitment threshold and a decrease in firing rate.

The present data differ in several important respects from previously-reported studies. The results reported by Garnett and Stephens $(1979,1981)$ and Datta and Stephens $(1979,1981)$ indicate that skin stimulation produces a different effect on recruitmēnt threshold and firing rate in the FDI, depending upon whether the motor unit is recruited above or below 1.5 N. In some of the subjects we tested, the maximum force of the FDI during index finger abduction was approximately $40 \mathrm{~N}$. Thus, the present data includes units with initial recruitment thresholds up to $45 \% \mathrm{MVC}$, in sharp contrast to the "high-threshold" units recorded at $4 \%$ MVC by Garnett and Stephens (1981). Among the six motor units with initial recruitment thresholds below 5\% MVC, two had a marked increase in recruitment threshold, but one decreased slightly, while the remaining three exhibited little change in the threshold for activation. In this lowforce range, our data indicate that the effect of the electrical stimulation is somewhat mixed, even among motor units of similar recruitment threshold. However, when one obtains an adequate sample size, it becomes clear that motor units with initial recruitment thresholds be- 
low 20\% MVC are predominantly inhibited by the electrical stimulation.

The idea that cutaneous receptors can exert differential effects on low- and high-threshold motor units is not altogether unexpected and is in agreement with previously-presented data (Burke 1981). The larger cutaneous afferents are most likely to be activated by the low-intensity electrical stimulation (Hallin and Torebjork 1973; Veltink et al. 1988), and these receptors tend to inhibit the smaller motoneurons while facilitating the larger motoneurons. However, the effect on all $\alpha$-motoneurons (especially those of mid-force range) tends to be mixed. Likewise, the data presented here demonstrates greater variability in recruitment threshold following skin stimulation than in control experiments (see Fig. 3), but there remains a tendency for the low-threshold units to be inhibited while high-threshold units are recruited at lower force levels.

In our preliminary experiments, we noted no effect produced by skin stimulation applied for five seconds in the midst of a 20-s contraction. We then changed to the present 3-minute stimulation protocol. Interestingly, Garnett and Stephens (1981) also observed a long time lag before an effect was observed. The mechanism for the long delay in the onset of these effects is not apparent, but one possibility may be that cutaneous afferents provide inputs to neuronal circuits that require considerable summation before an overt change in motoneuron excitability is observed. One example of such a circuit would be a reverberating loop (Hultborn et al. 1975). Alternatively, it may require many seconds for some humoral agent such as Substance P (Spanton and Prestwich 1981) to increase in concentration before a notable effect can be demonstrated.

The effect of digital nerve stimulation on FDI motor units seems to be similar to the effect of sural nerve stimulation on cat gastrocnemius and soleus muscles. Stimulation of sural nerve, for example, produces largely inhibitory post-synaptic potentials in MG motoneurons innervating slow twitch muscle fibers but largely excitatory post-synaptic potentials in motoneurons innervating fast-twitch muscle fibers (Burke et al. 1970). So in both cat and in man, cutaneous afferent input is not distributed in the same way to all motoneurons. While there may be some synaptic connections which are arranged at random or contrary to size principle, segmental or descending connections may override these other systems. Some recruitment reversals might occur among motor units that have unique synaptic connections with peripheral afferents. On occasion, some recruitment reversals may even occur without alterations in synaptic input (Henneman et al. 1976). The present results demonstrate that under conditions of increasing input from cutaneous receptors, net excitation to the motoneuron is altered such that the recruitment of high-threshold units can occur earlier, while the recruitment of low-threshold units is often delayed.

Acknowledgements. This work was conducted at the NeuroMuscular Research Center, Boston University. We would like to acknowledge the assistance of Messrs. Andrew Roy and Nanette Paul in these experiments. A special thanks is due Dr. Naoichi Chino,
Chairman and Associate Professor of Rehabilitation Medicine at Keio University School of Medicine, who afforded Dr. Masakado the opportunity to collaborate on this work. This research was supported by funds provided by the Liberty Mutual Insurance Company and the Department of Veterans Affairs, Rehabilitation Research and Development Service (B594-RA).

\section{References}

Binder MD, Mendell LM (1990) The segmental motor system. Oxford, New' York

Burke JR, Kamen G, Kocja DM (1989) Long-latency enhancement of quadriceps excitability from stimulation of skin afferents in young and old adults. J Gerontol 44:M158-M163

Burke RE (1973) On the central nervous system control of fast and slow twitch motor units. In: Desmedt JE (od) New developments in electromyography and clinical neurophysiology, Vol 3. Karger, Basel, pp 69-94

Burke RE (1981) Motor units : anatomy, physiology, and functional organization. In: Brooks VB (ed) Handbook of physiology, Sect 1. The nervous system, Vol II. Am Physiol Soc, Bethesda MD, pp 345-422

Burke RE, Jankowska E, ten Bruggencate G (1970) A comparison of peripheral and rubrospinal synaptic input to slow and fast twitch motor units of triceps surae. J Physiol (Lond) 207:709-732

Clamann HP, Gillies JD, Henneman E (1974) Effocts of inhibitory inputs on critical firing level and rank-order of motoneurons. J Neurophysiol 37:1350-1360

Clamann HP, Henneman E (1976) Electrical measurement of axon diameter and its use in relating motoneuron size to critical firing level. J Neurophysiol 39:844-856

Datta AK, Stephens JA (1979) The effect of digital nerve stimulation on motor unit interspike intervals recorded during voluntary contraction of the first dorsal interosseous muscle in man. J Physiol (Lond) 292: 16-17P

Datta AK, Stephens JA (1981) The effect of digital nerve stimulation on the firing of motor units in human first dorsal interosscous muscle. J Physiol (Lond) 318:501-510

Desmedt JE, Godaux E (1981) Spinal motoneuron recruitment in man: rank deordering with direction but not with speed of voluntary movement. Science 214:933-936

Garnett R, Stephens JA (1979) Changes in the recruitment threshold of motor units in human first dorsal interosscous muscle produced by skin stimulation. J Physiol (Lond) 282:13P

Garnett R, Stephens JA (1981) Changes in the recruitment threshold of motor units in human first dorsal interosseous muscle produced by skin stimulation. J Physiol (Lond) 311:463-473

Grimby L, Hannerz J (1976) Disturbances in voluntary recruitment order of low and high frequency motor units on blockades of proprioceptive afferent activity. Acta Physiol Scand 96:207-216

Hallin RG, Torebjork HE (1973) Electrically induced A and C fibre responses in intact human skin nerves. Exp Brain Res $16: 309-320$

Henneman E, Somjen G, Carpenter DC (1965) Functional significance of cell size in spinal motoneurons. J Neurophysiol 28:560-580

Henneman E, Shahani BT, Young RR (1976) Voluntary control of human motor units. In: Shahani $M$ (ed) The motor system: neurophysiology and muscle mechanisms. Elsevier, New York, pp 73-78

Hultborn H, Wigstrom H, Wangberg B (1975) Prolonged activation of soleus motoneurons following a conditioning train on soleus la afferents - a case for a reverberating loop? Neurosci Lett $1: 147-152$

Kanda K, Burke RE, Walmsley B (1977) Differential control of fast and slow twitch motor units in the decerebrate cat. Exp Brain Res 29:57-74

Kanda K, Desmedt JE (1983) Cutaneous facilitation oYarge motor units and motor control of human fingers in precision grip. In: 
Desmedt JE (cd) Motor control mechanisms in health and disease. Advances in neurology, Vol 29. Raven, New York, pp 253-261

LeFever RS, Xenakis AP, De Luca CJ (1982) A procedure for decomposing the myoelectric signal into its constituent action potentials, Part 2. Execution and test for accuracy. IEEE Trans Biomed Eng 29:158-164

Mambrito B, De Luca CJ (1983) Acquisition and decomposition of the EMG signal. In: Desmedt JE (ed) Computer-aided electromyography. Progress in clinical neurophysiology, Vol 10. Karger, Basel, pp 52-72

Mambrito B, De Luca CJ (1984) A technique for the detection, decomposition and analysis of the EMG signal. Electroencephal Clin Neurophysiol 58:175-188

McClean MD (1984) Recruitment threshold of lower-lip motor units with changes in movement diroction. J Spech Hearing Res 27:6-12

Mizote M (1982) The effect of digital nerve stimulation on recruitment order of motor units in the first deep lumbrical muscie of the cat. Brain Res 248:245-255

Nardone A, Romano C, Schieppati M (1989) Selective recruitment of high-threshold human motor units during voluntary isotonic lengthening of active muscles. J Physiol (Lond) 409:451-471

Person RS (1974) Rhythmic activity of a group of human motoneurones during voluntary contraction of a muscle. Electroencephal Clin Neurophysiol 36:585-595

Sabbahi MA, De Luca CJ (1982) Topical anesthesia: modulation of the monosynaptic reflexes by the desensitization of the skin. Electroencephal Clin Neurophysiol 54:677-688

Spanton SG, Prestwich GD (1981) Sensory and motor functions of spinal cord substance P. Science 214:1361-1364
Stashuk D, De Luca CJ (1989) Update on the decomposition and analysis of EMG signals In: Desmedt JE (ed) Computer-aided electromyography and expert systems. Clinical neurophysiology updates, Vol 2. Elsevier, New York, pp 39-53

Stein RB, Bertoldi R (1981) The size principle: a synthesis of neurophysiological data. In: Desmedt JE (ed) Motor unit types, recruitment and plasticity in health and disease. Progress in clinical neurophysiology, Vol 9. Karger, Basel, pp 85-96

Suzuki S, Hayami A, Suzuki M, Watanabe S, Hutton RS (1988) Alterations in human motor unit force thresholds by prior muscle activity and stretch. Med Science Sports Exerc 20 (Suppl):S54

Suzuki S, Hayami A, Suzuki M, Watanabe S, Hutton RS (1989) Successive muscle contractions decrease recruitment force thresholds in single motor units. Abstr Soc Neurosci 15:915

Suzuki S, Hayami A, Suzuki M, Watanabe S, Hutton RS (1990) Reductions in recruitment force thresholds in human single motor units by successive voluntary contractions. Exp Brain Res 82:227-230

Ter Haar Romeny BM, Denier JJ, Van der Gon CC, Gielen AM (1982) Changes in recruitment order of the motor units in the human biceps muscle. Exp Neurol 78:360-368

Thomas JS, Ross BH, Calancie B (1987) Human motor-unit recruitment during isometric contractions and repeated dynamic movements. J Neurophysiol 57:311-324

Thomas JS, Schmidt EM, Hambrecht FT (1978) Facility of motor unit control during tasks defined directly in terms of unit behaviors. Exp Neurol 59:384-395

Veltink PH, Van Alste JA, Boom HBK (1988) Influences of stimulation conditions on recruitment of myelinatod nerve fibers: a model study. IEE Trans Biomed Eng 35:917-924 